\title{
Mathematical Conditions in Heliostat Models for Deterministic Computation of Setpoints
}

\author{
Moisés Villegas-Vallecillos ${ }^{1}$ Luis J. Yebra ${ }^{2,3}$ \\ ${ }^{1}$ Departamento de Matemáticas, Universidad de Cádiz, Spain, moises.villegas @uca.es \\ ${ }^{2}$ Plataforma Solar de Almería, CIEMAT, Spain, luis.yebraepsa.es \\ ${ }^{3}$ CIESOL, Joint Centre of the University of Almería-CIEMAT, Spain
}

\begin{abstract}
In this paper a set of mathematical conditions on heliostat models is presented. Its purpose is to guarantee a deterministic computation of the heliostat setpoints in azimuth $(\beta)$ and elevation $(\alpha)$. In Central Receiver (CR) Concentrating Solar Power (CSP) plants, thousands of heliostats are continuously operated, and the updating of their setpoints is required frequently. For this reason, the fulfillment of some mathematical conditions of the mentioned type is important. In a simplified approach, during the operation, each heliostat reflects in its mirror a ray from the sun that impacts on a given aiming point $P$. This aiming point is assumed to be higher than the heliostat position, in the tower receiver. If $v$ is the incident solar vector, $x$ is the orthogonal vector of the heliostat reflective plane and $f(x)$ is the center of the heliostat mirror, then a system of equations with unknown $x$ is arisen. Imposing certain conditions on $f$, we can ensure the existence and uniqueness of solution of this system, and provide a sequence converging to such solution. Furthermore, we offer a numerical method for approximating the solution in a deterministic form, which can be computed with the requirements of hard real time systems.

Keywords: central receiver concentrating solar thermal plants, heliostat setpoint, Banach's fixed point theorem, Newton-Raphson's numerical method, deterministic computation
\end{abstract}

\section{Introduction}

From a simplified point of view, a heliostat could be considered as a device that supports a mirror and orientates it, following the daily motion of the sun through a servomechanism and a multibody system (Otter et al., 2003). The mirror should be continuously properly oriented to reflect the sun radiation into an aiming point (objective point) positioned in the receiver of a Central Receiver (CR) Concentrating Solar Power (CSP) plant. In this way, all the heliostats in a CR CSP plant will concentrate the primary power from the sun into a set of predefined aiming points, that are carefully selected to generate a distributed power density pattern in the receiver. Figures 1 and 2 show this scenario.

Some authors have studied different ways to improve

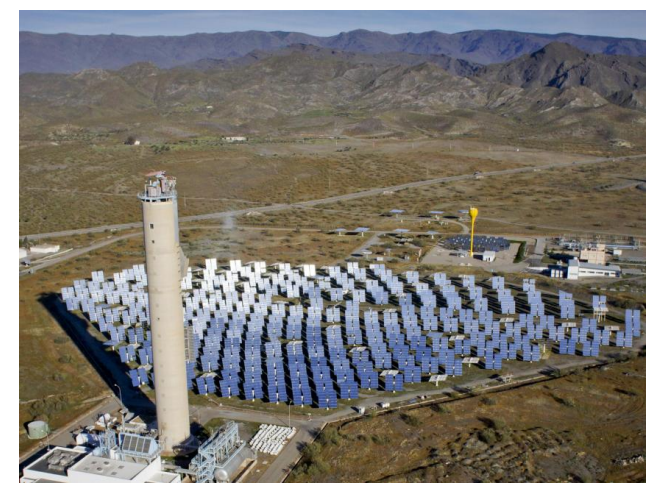

Figure 1. CESA-I central receiver research facility at Plataforma Solar de Almería (PSA).

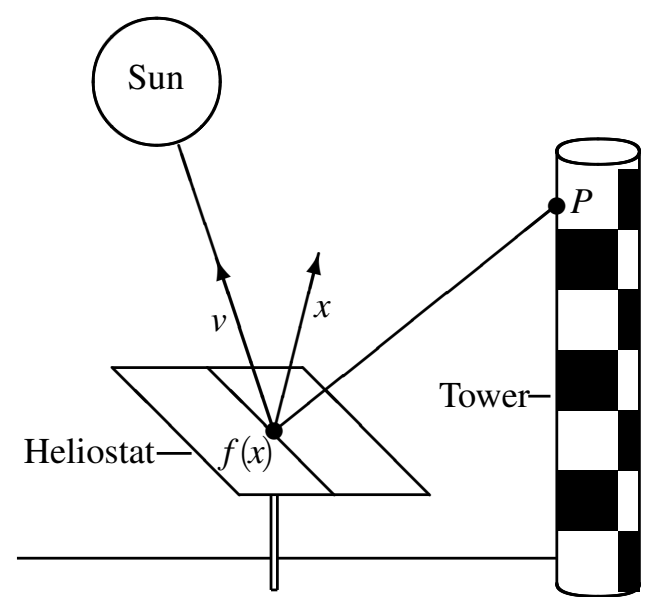

Figure 2. Simplified scheme showing the reflection of a ray representing the direct component of solar radiation being reflected in the heliostat mirror and impacting in one of the aiming points $P$ of the receiver.

the focusing system of the heliostats. For example, Mulholland presents in (Mulholland, 1983) a method to determine the optimum size of the heliostat or concentrator, Guo et al. give in (Guo et al., 2012) a least squares model to determine certain angular errors in some altitudeazimuth tracking formulas, García-Martín et al. develop in (García-Martín et al., 1999) a control strategy aimed at optimizing the temperature distribution within a volumetric receiver, Berenguel et al. apply in (Berenguel et al., 
2004) a correction control system using artificial vision techniques, etc.

A related issue is to properly distribute the reception points of the tower where solar energy is focused. So it will be possible to get a uniform temperature profile in the receiver and prevent its structure from being damaged. To achieve that and develop a robust control system for the heliostat field, the following problem is considered:

Given a heliostat in which the center $C$ and the orthogonal vector $x$ of its mirror plane are variable, how can be determined them so that the reflected ray impacts on a specific receiver point $P$ ?

When the center $C$ of the heliostat mirror can be expressed as a function $f(x)$ of the orthogonal vector $x$, it is possible to raise a system of equations to determine $x$ (Section 2). In such case, some conditions are given on $f$ to ensure the existence and uniqueness of solution of such system, and to obtain a sequence converging to that solution (Section 3). Concretely, it is required that $f$ is a Lipschitz function and its Lipschitz constant verifies certain boundedness (see Condition 3.3).

Furthemore, a numerical method is developed to approximate the solution by mixing the aforementioned convergent sequence with Newton-Raphson's method. Thus, a safe and good speed convergence is obtained (Sections 4 and 5), making the numerical method a potential solution to use in hard real time control systems (Burns and Wellings, 2010).

\section{Approach to the system of equations}

In principle, it must be considered the relationship between the incident ray, the reflected ray and the reflective surface of the heliostat. That relationship is given by the law of reflection (Hecht, 2002, pages 98 and 99).

Law 2.1 (The Law of Reflection) The incident ray, the normal to the reflective surface and the reflected ray all lie in the same plane. Furthermore, the angle of reflection is equal to the angle of incidence.

This law and Proposition 2.3 are key tools to raise a system of equations. Before stating that proposition, a lemma is presented that will simplify its proof. Throughout all this paper, $(\cdot \mid \cdot)$ will denote the usual Euclidean inner product of $\mathbb{R}^{N}$ (where $N$ is some positive integer), and $\|\cdot\|$ will represent the norm induced by this inner product. On the other hand, given $v_{1}, \ldots, v_{m} \in \mathbb{R}^{N}$, we denote by $\operatorname{lin}\left(\left\{v_{1}, \ldots, v_{m}\right\}\right)$ the linear subspace spanned by $\left\{v_{1}, \ldots, v_{m}\right\}$ (see (Larson and Falvo, 2012) for details).

Lemma 2.2 Let $u, w \in \mathbb{R}^{N}$ vectors with $\|u\|=1=\|w\|$ and $u \neq-w$. If $\theta$ is the angle formed by the vectors $u$ and $w$, then the angle between $u$ and $u+w$ is $\theta / 2$. As a consequence, $(u \mid u+w) \geq 0$.

Next, the announced key proposition is presented.
Proposition 2.3 Let $v, P, C \in \mathbb{R}^{3}$ such that $v$ and $P-C$ are linearly independent, and let $x=P-C+(\|P-C\| /\|v\|) v$. It is satisfied that:

i) If the heliostat reflective plane $H$ passes through the point $C$ and has orthogonal vector $x$, then the plane $C+\operatorname{lin}(\{v, x, P-C\})$ is perpendicular to $H$.

ii) The angle between $P-C$ and $x$ is equal to the angle between $v$ and $x$.

iii) The vectors $v$ and $P-C$ are in the half-space determined by $x$, that is, $0 \leq(v \mid x)$ and $0 \leq(P-C \mid x)$.

If the incident solar ray passes through the point $C$ and has direction vector $v$, and the heliostat reflective plane $H$ passes through $C$ and has orthogonal vector

$$
x=P-C+(\|P-C\| /\|v\|) v
$$

then this proposition and the law of reflection state that the reflected ray passes through $C$ and $P$.

It is not possible that $v$ and $P-C$ are linearly dependent because the tower where $P$ is situated prevents (with its shadow) the existence of an incident solar ray passing through $P$ and $C$. But even if $v$ and $P-C$ are linearly dependent and there exits the incident solar ray, the reflected ray and the line through $C$ and $P$ are coincident when $H$ has orthogonal vector $x=P-C+(\|P-C\| /\|v\|) v$ (in fact, they would also coincide with the incident ray and the normal to $H$ ). Therefore, it will not be necessary to suppose that $v$ and $P-C$ are linearly independent in subsequent developments.

Conditions 2.4 From now on we assume the following conditions given by the nature of the problem:

a) The director vector $v=\left(a_{1}, a_{2}, a_{3}\right)$ of the incident solar ray satisfies that $a_{3} \geq 0$.

b) We suppose that the center $C$ of the heliostat mirror can be expressed as a function of its orthogonal vector, that is, there exists a function $f: \mathbb{R}^{3} \backslash\{0\} \rightarrow \mathbb{R}^{3}$ such that, if $x \in \mathbb{R}^{3} \backslash\{0\}$ is a orthogonal vector to the heliostat mirror, then $C=f(x)$ is the center of such mirror. Note that, in such case, it holds $f(r x)=f(x)$ for all $x \in \mathbb{R}^{3} \backslash\{0\}$ and for all $r>0$.

c) Whatever the center of the heliostat mirror, the distance from it to the impact point of the tower is always less than certain upper bound, that is, there is $R>0$ such that $\|P-f(x)\| \leq R$ for all $x \in \mathbb{R}^{3} \backslash\{0\}$.

d) The impact point of the tower $P=\left(p_{1}, p_{2}, p_{3}\right)$ is quite higher than the heliostat, so there is a lower bound $M>0$ such that $M \leq p_{3}-f_{3}(x)$ for all $x \in \mathbb{R}^{3} \backslash\{0\}$.

Moreover, we consider the following subset of $\mathbb{R}^{3}$ :

$$
D_{M}=\left\{\left(x_{1}, x_{2}, x_{3}\right) \in \mathbb{R}^{3}: M \leq x_{3},\left\|\left(x_{1}, x_{2}, x_{3}\right)\right\| \leq 2 R\right\} .
$$


Taking into account Proposition 2.3 and the comments following it, if there exists $x \in \mathbb{R}^{3} \backslash\{0\}$ such that

$$
x=P-f(x)+\frac{\|P-f(x)\|}{\|v\|} v
$$

and the heliostat reflective plane is orthogonal to $x$, then the ray reflected by the heliostat impacts on the point $P$. Therefore, we must solve the system of equations given by equality (2). Fortunately, we do not have to require too much of $f$ to ensure the existence of solution of system (2). This is shown by the following result (which can be found in (Deimling, 1985, Theorem 3.2)).

Theorem 2.5 (Brouwer's Fixed Point Theorem) Let D be a nonempty compact convex subset of $\mathbb{R}^{N}$ and let $g: D \rightarrow D$ be a continuous function. Then $g$ has a fixed point, that is, there exists $x \in D$ with $x=g(x)$. The same is true if $D$ is only homeomorphic to a compact convex set.

Since $P-f(x)+(\|P-f(x)\| /\|v\|) v \in D_{M}$ for all $x \in D_{M}$, then Theorem 2.5 shows that system (2) has solution in $D_{M}$ when it is assumed

Condition 2.6 $f$ is continuous on $D_{M}$.

\section{Sufficient conditions for uniqueness of solution and convergence}

Before continuing with our development, we recall some concepts and an important result. Given $D \subseteq \mathbb{R}^{N}$ and a function $g: D \rightarrow \mathbb{R}^{N}$, it is said that $g$ is Lipschitz on a subset $A \subseteq D$ if there is a real constant $c \geq 0$ such that

$$
\|g(x)-g(y)\| \leq c\|x-y\|
$$

for all $x, y \in A$. The least constant $c$ for which the preceding inequality holds will be denoted by $L\left(\left.g\right|_{A}\right)$. If $L\left(\left.g\right|_{A}\right)<1$, it is said that $g$ is a contraction mapping on $A$.

A basic tool to prove existence and uniqueness of solutions of systems of equations is Banach's celebrated fixed-point theorem. It is true for general complete metric spaces but here we only need its version for closed subsets of $\mathbb{R}^{N}$ (see (Deimling, 1985, Theorem 7.1) or (Edwards, 1994, Theorem 3.1)).

Theorem 3.1 (Banach's Fixed Point Theorem) Let $D$ be a closed subset of $\mathbb{R}^{N}$ and $g: D \rightarrow D$ be a contraction mapping on D. Then:

i) g has a unique fixed point $x \in D$.

ii) For all $x^{(0)} \in D$, the sequence $\left\{x^{(n)}\right\}$, given by

$$
x^{(n+1)}=g\left(x^{(n)}\right) \quad(n \in \mathbb{N} \cup\{0\}),
$$

converges to $x$, and satisfy

$$
\left\|x^{(n)}-x\right\| \leq \frac{L(g)^{n}}{1-L(g)}\left\|x^{(1)}-x^{(0)}\right\| \quad(n \in \mathbb{N}) .
$$

Our aim in this section is to obtain a sequence converging to a solution of system (2) by using Theorem 3.1. In the following proposition we give sufficient conditions on $f$ to get a contraction mapping and be able to apply Theorem 3.1.

Proposition 3.2 Let $v=\left(a_{1}, a_{2}, a_{3}\right), P=\left(p_{1}, p_{2}, p_{3}\right) \in \mathbb{R}^{3}$ be with $a_{3} \geq 0, M, R \in \mathbb{R}$ be bounds with $0<M<R$ and $f: \mathbb{R}^{3} \backslash\{0\} \rightarrow \mathbb{R}^{3}$ be a function such that $M \leq p_{3}-f_{3}(x)$, $\|P-f(x)\| \leq R$ and $f(r x)=f(x)$ for all $x \in \mathbb{R}^{3} \backslash\{0\}$ and for all $r>0$. Suppose that there exists $\delta \in(0, M]$ such that $f$ is Lipschitz on the set

$$
D_{\delta}=\left\{\left(x_{1}, x_{2}, x_{3}\right) \in \mathbb{R}^{3}: \delta \leq x_{3},\left\|\left(x_{1}, x_{2}, x_{3}\right)\right\| \leq 2 R\right\}
$$

and $L\left(\left.f\right|_{D_{\delta}}\right)<M /(2 \delta)$. Then the function $g: D_{M} \rightarrow D_{M}$, defined by

$$
g(x)=P-f(x)+\frac{\|P-f(x)\|}{\|v\|} v \quad\left(x \in D_{M}\right),
$$

is a contraction mapping on $D_{M}$ and $L(g) \leq 2 \delta L\left(\left.f\right|_{D_{\delta}}\right) / M$.

The new condition given by Proposition 3.2 is

Condition 3.3 There exists $\delta \in(0, M]$ such that $f$ is Lipschitz on $D_{\delta}$ and $L\left(\left.f\right|_{D_{\delta}}\right)<M /(2 \delta)$.

If we assume this condition, Theorem 3.1 and Proposition 3.2 tell us that system (2) has a unique solution in $D_{M}$, and provide us with a sequence converging to that solution.

Remark 3.4 Supposing that the function $f$ is Lipschitz on $D_{\delta}$ is not too restrictive. For example, if we assume that $f$ is of class $\mathscr{C}^{1}$ on some open set containing $D_{\delta}$, then $f$ is Lipschitz on $D_{\delta}$. We give a bound for the Lipschitz constant of $f$ in this situation. As the partial derivatives of $f$ are bounded on $D_{\delta}$, we can find $k_{1}(\boldsymbol{\delta}), k_{2}(\boldsymbol{\delta}), k_{3}(\boldsymbol{\delta}) \in$ $\mathbb{R}_{0}^{+}$such that

$$
\left\|\nabla f_{j}(x)\right\| \leq k_{j}(\delta) \quad\left(x \in D_{\delta}, j=1,2,3\right) .
$$

It can be proved by applying the Mean Value Theorem (Edwards, 1994, Theorem 3.4) that the Condition 3.3 is fulfilled if

$$
k_{1}(\delta)+k_{2}(\delta)+k_{3}(\delta) \leq M /(2 \delta) .
$$

\section{Newton-Raphson's method for the raised system}

In this section we recall Newton-Raphson's method for solving system (2). Suppose we want to solve a system of three equations with three unknowns of the form $F(x)=0$, where $F$ is a function from a subset $\Omega \subseteq \mathbb{R}^{3}$ into $\mathbb{R}^{3}$. One idea is to find a matrix $Q=\left(q_{i j}\right)$ whose entries $q_{i j}$ are functions from $\Omega$ into $\mathbb{R}$, such that the fixed-point iteration determined by the function $G: \Omega \rightarrow \mathbb{R}^{3}$, where

$$
G(x)=x-Q(x) F(x) \quad(x \in \Omega) ;
$$


gives quadratic convergence to the solution of $F(x)=0$. The following theorem motivates the choice of $Q$ (see (Burden and Faires, 2010, Theorem 10.7)). Its proof is based on the Taylor development of $G$ (consult (Edwards, 1994, Theorem 7.1)).

Theorem 4.1 Let $\Omega$ be an open subset of $\mathbb{R}^{N}, A \subseteq \Omega$ be a convex subset and $G: \Omega \rightarrow \mathbb{R}^{N}$ be a function of class $\mathscr{C}^{2}$ on $\Omega$. Suppose that there exists a fixed point $w \in A$ of $G$, and $G$ has the following properties:

a) $\left|\partial^{2} G_{i}(x) /\left(\partial x_{j} \partial x_{k}\right)\right| \leq K$ for each $x \in A, i, j, k \in$ $\{1, \ldots, N\}$, and for some constant $K>0$ (that is, the second partial derivatives of $G$ are bounded on $A$ ).

b) $\partial G_{i}(w) / \partial x_{j}=0$ for all $i, j \in\{1, \ldots, N\}$.

Then, for all $x^{(0)} \in A$ with $\left\|x^{(0)}-w\right\|<2 /\left(N^{5 / 2} K\right)$, the sequence generated by

$$
x^{(n+1)}=G\left(x^{(n)}\right) \quad(n \in \mathbb{N} \cup\{0\})
$$

converges quadratically to w. Concretely,

$$
\left\|x^{(n+1)}-w\right\| \leq \frac{N^{5 / 2} K}{2}\left\|x^{(n)}-w\right\|^{2} \quad(n \in \mathbb{N} \cup\{0\}) .
$$

When $G(x)=x-Q(x) F(x)$, and $Q, F$ are of class $\mathscr{C}^{1}$, the second condition of Theorem 4.1 is equivalent to $Q(w) J_{F}(w)=I_{3}$, where $J_{F}(w)$ is the Jacobian matrix of $F$ at the point $w$ and $I_{3}$ is the identity matrix. So NewtonRaphson's method consists in choosing $Q(x)=J_{F}(x)^{-1}$ for each $x \in \Omega$, and performing the fixed point iteration determined by the function $G$, where

$$
G(x)=x-J_{F}(x)^{-1} F(x) \quad(x \in \Omega) .
$$

Remark 4.2 To apply Newton-Raphson's method and ensure its quadratic convergence to the solution of the system (using Theorem 4.1), we must impose the following conditions:

i) $\Omega$ must be an open neighbourhood of the solution $w$ of the system $F(x)=0$.

ii) F has to be of class $\mathscr{C}^{3}$ on $\Omega$ (take in mind the inverse mapping theorem).

iii) $\operatorname{det}\left(J_{F}(x)\right) \neq 0$ for all $x \in \Omega$.

iv) The second partial derivatives of the function $G$ must be bounded on a convex subset $A \subseteq \Omega$ containing $w$.

v) The first value of the iteration $x^{(0)} \in A$ has to be close enough to the solution $w$.

Next we see how are these requirements in our context. Let $f: \mathbb{R}^{3} \backslash\{0\} \rightarrow \mathbb{R}^{3}, v, P \in \mathbb{R}^{3}$ and $R, M \in \mathbb{R}$ be as in Conditions 2.4 , and let

$$
D_{M}=\left\{\left(x_{1}, x_{2}, x_{3}\right) \in \mathbb{R}^{3}: M \leq x_{3},\left\|\left(x_{1}, x_{2}, x_{3}\right)\right\| \leq 2 R\right\} .
$$

Consider the function $F: \mathbb{R}^{3} \backslash\{0\} \rightarrow \mathbb{R}^{3}$ defined by

$$
F(x)=P-f(x)+\frac{\|P-f(x)\|}{\|v\|} v-x \quad\left(x \in \mathbb{R}^{3} \backslash\{0\}\right) .
$$

Conditions 4.3 The requisites above can be rewritten for system (2) as follows:

a) $f$ must be of class $\mathscr{C}^{3}$ on an open subset $\Omega \subseteq \mathbb{R}^{3} \backslash\{0\}$ containing $D_{M}$.

b) $\operatorname{det}\left(J_{F}(x)\right) \neq 0$ for all $x \in \Omega$.

c) To perform the fixed point iteration given by the function $G: \Omega \rightarrow \mathbb{R}^{3}$, where $G(x)=x-J_{F}(x)^{-1} F(x)$ for all $x \in \Omega$, the first value $x^{(0)} \in D_{M}$ has to be close enough to the solution.

Note that under these requirements, as $D_{M}$ is compact and $G$ is of class $\mathscr{C}^{2}$, it holds that the second partial derivatives of $G$ are bounded on $D_{M}$.

\section{A numerical method to approximate the solution}

Under the conditions of Proposition 3.2, the development carried out in Section 3 gives us an iterative method to approximate the solution of system (2). This is the fixed point iteration given by the contraction mapping $g$. The convergence of this method is safe. However, the method converges with linear speed, whence it is slower than Newton-Raphson's method.

On the other hand, when $f$ is of class $\mathscr{C}^{3}$ on an open subset containing $D_{M}$, we have no guarantees that Newton-Raphson's method can be applied because, a priori, we do not know if $\operatorname{det}\left(J_{F}(x)\right) \neq 0$. Moreover the convergence of Newton-Raphson's method is local, since $x^{(0)}$ must be chosen close enough to the solution.

For these reasons, we try to use both methods together in order to take advantage of both. First, we get close to the solution using the contraction mapping method. We must set an initial tolerance tol0, and, when it is attained, we change over to Newton-Raphson's method. If Newton-Raphson's method can not be applied, we continue with the contraction mapping method. In Algorithm 1 we can see the procedure.

Note that, to apply this algorithm, the function $f$ of the heliostat and Newton-Raphson's method must first be implemented. An outline of Newton-Raphson's method can be found in (Burden and Faires, 2010, Algorithm 10.1). To do its implementation, take into account that the method fails when the Jacobian matrix of $F$ is not regular, there is no convergence $\left(2\right.$ tol $\left.0 \leq\left\|x^{(k+1)}-x^{(k)}\right\|\right)$ or the maximum number of iterations is exceeded. 


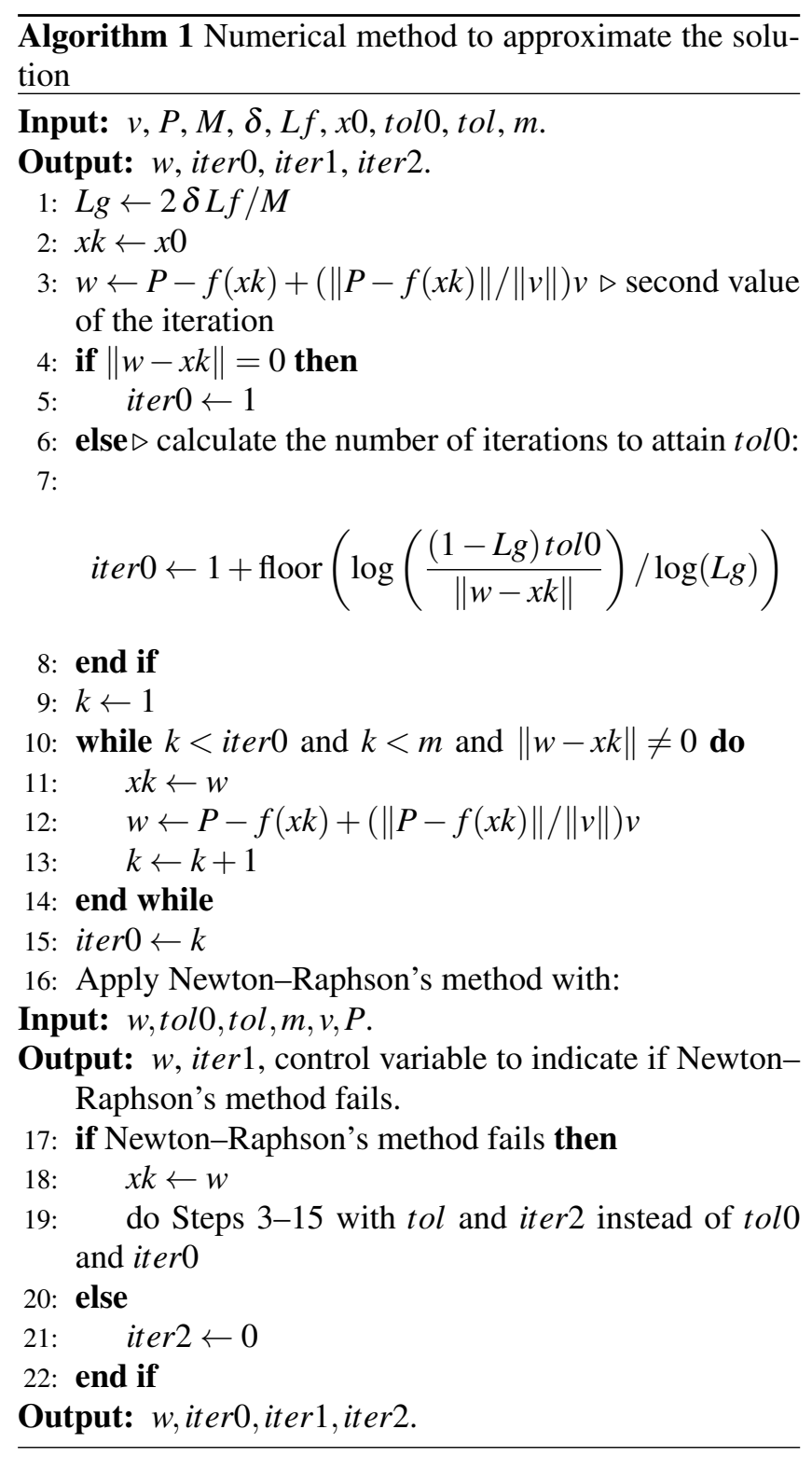

\section{First example. Type B heliostats}

In type B heliostats there is a unique joint between two pieces: the post and the arm (see Fig. 3). The heliostat mirror is at the end of the arm so that the mirror plane is perpendicular to the arm. Let $Q$ be the point where the joint is situated and $l$ be the length of the arm. If $x$ is a vector orthogonal to the mirror plane, then the center of the heliostat mirror is given by

$$
f(x)=Q+\frac{l}{\|x\|} x
$$

As $f$ is continuous, Theorem 2.5 ensures the existence of solution of system (2). In fact, given $\delta \in(0, M], f$ is Lipschitz on $D_{\delta}$ and $L\left(\left.f\right|_{D_{\delta}}\right) \leq 2 l / \delta$. Then Proposition 3.2 can be applied if it is satisfied that $2 l / \delta<M /(2 \delta)$ for some $\delta \in(0, M]$. Taking, for example, $\delta=M$, this inequality is fulfilled since $4 l<M$. Therefore Proposition 3.2 and Theorem 3.1 give us existence and uniqueness

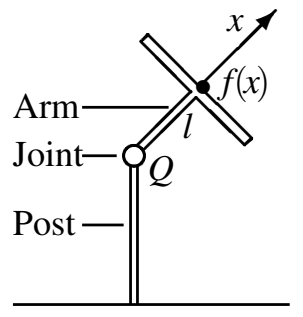

Figure 3. Type B heliostat

of solution of the system (2) on $D_{M}$, and a fix point iteration converging to it. Moreover $L\left(\left.f\right|_{D_{M}}\right) \leq 2 l / M$ and $L(g) \leq 4 l / M$.

Besides, to apply Newton-Raphson's method, we need the partial derivatives of the function $F: \mathbb{R}^{3} \backslash\{0\} \rightarrow \mathbb{R}^{3}$ given, for each $x \in \mathbb{R}^{3} \backslash\{0\}$, by

$$
F(x)=P-Q-\frac{l}{\|x\|} x+\left\|P-Q-\frac{l}{\|x\|} x\right\| \frac{v}{\|v\|}-x .
$$

We prove straightforwardly that the Jacobian matrix of $F$ is

$$
\begin{aligned}
J_{F}(x)= & \frac{l}{\|x\|}\left(\frac{x x^{T}}{\|x\|^{2}}-I_{3}\right) \\
& +\frac{l v\left(P-Q-\frac{l}{\|x\|} x\right)^{T}}{\|x\|\|v\|\left\|P-Q-\frac{l}{\|x\|} x\right\|}\left(\frac{x x^{T}}{\|x\|^{2}}-I_{3}\right)-I_{3}
\end{aligned}
$$

where the vectors are considered as $3 \times 1$ column matrices and $I_{3}$ is the identity matrix.

Example 6.1 Consider two type B heliostats, one with its joint at the point $Q_{1}=(-0.008,186.996,5.445)$ and other with its joint at $Q_{2}=(-35.191,383.648,11.125)$. Both heliostats have an arm of a meter in length. Two solar rays can strike on them. Their direction vectors are $v_{1}=(0.591431,-0.164578,0.789382)$ and $v_{2}=$ $(0.510816,-0.199895,0.836127)$. It is desired that, for both solar incident rays, the reflected rays from each heliostat impact first on the point $P_{1}=(20,1.7,75)$, after on the point $P_{2}=(-0.6,1.738,73.991)$ and finally on $P_{3}=$ $(0.008,6.524,34.165)$ (all coordinates are in meters). We can take the bound $M=34-13=21$. The obtained results are shown in Table 1. Remember that our numerical method (Algorithm 1) consists of three stages. In Table 1, the number of iterations of the first stage (fixed point iteration of the function $g$ ) is represented by FP, for the second stage (Newton-Raphson's method) we put NR, and for the last stage (again fixed point iteration of the function $g$ ) FP is used too.

Finally, we compare the hybrid method and NewtonRaphson's method. Taking the first heliostat, the incident solar vector $v_{1}$ and the impact point $P_{1}$, then the solution $x=(136.7502,-217.2572,225.2455)$ is obtained 
Table 1. Results with type B heliostats

\begin{tabular}{c|c|c|c|c|c}
\hline$x_{1}$ & $x_{2}$ & $x_{3}$ & FP & NR & FP \\
\hline \hline 136.7502 & -217.2572 & 225.2455 & 4 & 2 & 0 \\
\hline 115.3882 & -216.9750 & 223.1364 & 4 & 2 & 0 \\
\hline 107.2535 & -209.6961 & 171.7516 & 4 & 2 & 0 \\
\hline 120.8285 & -224.2384 & 234.4766 & 4 & 2 & 0 \\
\hline 99.5775 & -223.9091 & 232.3027 & 4 & 2 & 0 \\
\hline 92.6350 & -216.1090 & 180.2265 & 4 & 2 & 0 \\
\hline 285.6065 & -445.4998 & 371.4253 & 5 & 1 & 0 \\
\hline 263.5169 & -445.0280 & 368.3868 & 5 & 1 & 0 \\
\hline 258.7250 & -438.7159 & 321.4195 & 4 & 2 & 0 \\
\hline 254.1878 & -459.2700 & 389.6425 & 5 & 1 & 0 \\
\hline 232.3052 & -458.7087 & 386.4839 & 5 & 1 & 0 \\
\hline 228.2491 & -452.0741 & 339.0899 & 4 & 2 & 0 \\
\hline
\end{tabular}

Table 2. Comparison of Hybrid and Newton-Raphson's methods

\begin{tabular}{c||c||c||c}
\hline & iter0 & iter1 & iter2 \\
\hline \hline Hybrid & 4 & 2 & 0 \\
\hline N.R. & & 5 & \\
\hline
\end{tabular}

with both methods. However the number of iterations employed by each method changes. This is shown in Table 2 . The columns iter 0 , iter 1 and iter 2 represent the number of iterations in each of the stages of the hybrid method as detailed in Algorithm 1. For Newton-Raphson's method, iter 1 correspond to the total number of effected iterations.

The iterations of Newton-Raphson's method are fewer, but we must observe that:

1. Once the first iterations of the hybrid method are passed, it is also used Newton-Raphson's method.

2. At each step of Newton-Raphson's method a Jacobian matrix must be calculate and a linear system must be solved. Hence the number of operations of Newton-Raphson's method is greater than the number of operations of the fixed point iteration given by the contraction mapping $g$.

3. There is no guarantees that Newton-Raphson's method can be always applied.

\section{Second example. Type A heliostats}

In this section, we consider the simplest heliostat type. Now the center of the mirror is fixed, that is, there is $C=\left(c_{1}, c_{2}, c_{3}\right) \in \mathbb{R}^{3}$ such that $f(x)=C$ for all $x \in \mathbb{R}^{3} \backslash\{0\}$. Then system (2) is trivially solved without applying any

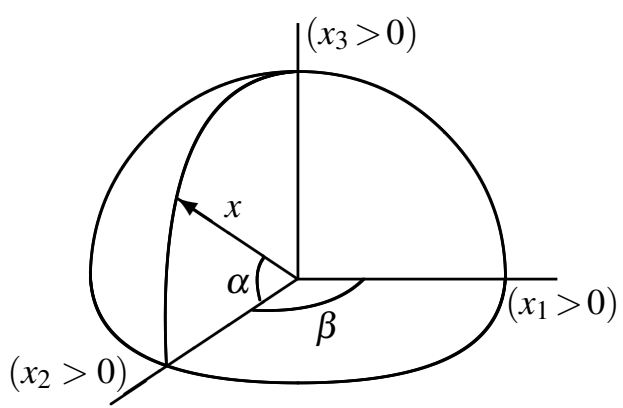

Figure 4. Spherical coordinates

numerical method because we have

$$
x=P-C+\frac{\|P-C\|}{\|v\|} v .
$$

It may be that a spherical coordinates of the orthogonal vector $x$ are needed. For example, the angles $\alpha \in$ $[-\pi / 2, \pi / 2]$ and $\beta \in(-\pi, \pi]$ determined by Fig. 4 may be required. In the following example these angles are calculated.

Example 7.1 A simple heliostat has the center of its mirror at the point $C_{1}=(-0.008,186.996,5.445)$, and another has it at the point $C_{2}=(-35.191,383.648,11.125)$. Two solar rays can strike on them, one with direction vector $v_{1}=(0.591431,-0.164578,0.789382)$ and other with direction vector $v_{2}=(0.510816,-0.199895,0.836127)$. It is desired that, for both solar incident rays, the reflected rays from each heliostat impact on the points $P_{1}=(20,1.7,75)$, $P_{2}=(-0.6,1.738,73.991)$ and $P_{3}=(0.008,6.524,34.165)$ (all coordinates are in meters). The implemented computations give the results presented in Table 3 .

\section{Conclusions}

If a heliostat meets that the center $C$ of its mirror can be expressed as a function $f(x)$ of its orthogonal vector $x$, and such function $f$ satisfies certain general conditions (condition 3.2), then, given an incident solar vector $v$ and a specific receiver point $P$, there exists $x$ making the reflected ray impacts on $P$. Furthermore, a numerical method is developed to approximate the solution $x$, with safe and good speed convergence properties. These properties make the numerical method a potential solution to use in hard real time control systems for the deterministic computation of the heliostat setpoints.

As we do not work with a specific function $f$, the model proposed to find the orthogonal vector $x$ can be applied to different types of heliostats. For each heliostat type a mathematical model should be derived to obtain the 
Table 3. Results with type A heliostats

\begin{tabular}{c|c|c|c|c}
\hline $\begin{array}{c}\text { Center } \\
\text { of } \\
\text { heliostat } \\
\text { mirror }\end{array}$ & $\begin{array}{c}\text { Incident } \\
\text { solar } \\
\text { vector }\end{array}$ & $\begin{array}{c}\text { Impact } \\
\text { point } \\
\text { of the } \\
\text { tower }\end{array}$ & $\begin{array}{c}\text { Angle } \alpha \\
\text { (radians) }\end{array}$ & $\begin{array}{c}\text { Angle } \beta \\
\text { (radians) }\end{array}$ \\
\hline \hline$C_{1}$ & $v_{1}$ & $P_{1}$ & 0.72094 & -1.0076 \\
\hline$C_{1}$ & $v_{1}$ & $P_{2}$ & 0.73797 & -1.0805 \\
\hline$C_{1}$ & $v_{1}$ & $P_{3}$ & 0.63113 & -1.0965 \\
\hline$C_{1}$ & $v_{2}$ & $P_{1}$ & 0.74487 & -1.0753 \\
\hline$C_{1}$ & $v_{2}$ & $P_{2}$ & 0.75955 & -1.1510 \\
\hline$C_{1}$ & $v_{2}$ & $P_{3}$ & 0.65516 & -1.1645 \\
\hline$C_{2}$ & $v_{1}$ & $P_{1}$ & 0.61247 & -1.0001 \\
\hline$C_{2}$ & $v_{1}$ & $P_{2}$ & 0.61942 & -1.0355 \\
\hline$C_{2}$ & $v_{1}$ & $P_{3}$ & 0.56352 & -1.0373 \\
\hline$C_{2}$ & $v_{2}$ & $P_{1}$ & 0.63908 & -1.0647 \\
\hline$C_{2}$ & $v_{2}$ & $P_{2}$ & 0.64510 & -1.1014 \\
\hline$C_{2}$ & $v_{2}$ & $P_{3}$ & 0.59063 & -1.1027 \\
\hline
\end{tabular}

function $f$.

\section{Acknowledgment}

The authors gratefully acknowledge the funding support from CIEMAT Research Centre, EU $7^{\text {th }}$ Framework Programme (Theme Energy 2012.2.5.2) under grant agreement 308912 - HYSOL project - Innovative Configuration of a Fully Renewable Hybrid CSP Plant, the National R+D+i Plan Project DPI2014-56364-C2-2-R of the Spanish Ministry of Economy and Competitiveness and ERDF funds.

\section{Basic symbols}

$\|\cdot\|:$ usual Euclidean norm of $\mathbb{R}^{N}$.

$v$ : incident solar vector.

$P$ : aiming point in the tower receiver.

$C$ : center of the heliostat mirror.

$x$ : orthogonal vector of the heliostat mirror plane.

$f$ : function relating the orthogonal vector $x$ and the center

$C$ of the heliostat mirror.

$R$ : maximum distance between $P$ and the center of the heliostat mirror (or greater).

$M$ : minimum difference between the height of $P$ and the height of the heliostat mirror center (or less).

$\delta$ : a positive constant in the interval $(0, M]$.
$D_{\delta}$ : set of vectors in the closed ball of center 0 and radius $2 R$ whose third coordinate is greater than or equal to $\delta$.

$L\left(\left.g\right|_{A}\right)$ : Lipschitz constant of the function $g$ on the set $A$ (when $A$ is the domain of the function $g$, we put $L(g)$ ). $g$ : function given by $g(x)=P-f(x)+(\|P-f(x)\| /\|v\|) v$. $\nabla f_{j}$ : gradient of the function $f_{j}$.

\section{References}

M. Berenguel, F. R. Rubio, A. Valverde, P. J. Lara, M. R. Arahal, E. F. Camacho, and M. López. An artificial vision-based control system for automatic heliostat positioning offset correction in a central receiver solar power plant. Solar Energy, 76: 563-575, 2004.

R. L. Burden and J. D. Faires. Numerical analysis. Brooks Cole, Boston, 9th edition, 2010.

A. Burns and A. J. Wellings. Real-time systems and programming languages, volume 2097. Addison-Wesley, 2010.

K. Deimling. Nonlinear Functional Analysis. SpringerVerlag, Berlin, 1985.

C. H. Edwards. Advanced Calculus of Several Variables. Dover books on advanced mathematics. Dover Publications, revised edition, 1994.

F. J. García-Martín, M. Berenguel, A. Valverde, and E. F. Camacho. Heuristic knowledge-based heliostat field control for the optimization of the temperature distribution in a volumetric receiver. Solar Energy, 66 (5): 355-369, 1999.

M. Guo, Z. Wang, J. Zhang, F. Sun, and X. Zhang. Determination of the angular parameters in the general altitude-azimuth tracking angle formulas for a heliostat with a mirror-pivot offset based on experimental tracking data. Solar Energy, 86 (3): 941-950, 2012.

E. Hecht. Optics. Addison-Wesley, New York, 4th edition, 2002.

R. Larson and D. Falvo. Elementary Linear Algebra. Belmont, CA: Brooks/Cole Cengage Learning, 7th international edition, 2012.

G. P. Mulholland. Determination of heliostat and concentrator size for solar furnace facilities. Journal of Solar Energy Engineering, 105 (3): 243-245, 1983.

M. Otter, H. Elmqvist, and S. E. Mattsson. The new modelica multibody library. In Proceedings of the 3rd International Modelica Conference. Citeseer, 2003. 